\title{
Custos e escala de produção na pecuária leiteira: estudo nos principais estados produtores do Brasil
}

\author{
Patrick Fernandes Lopes ${ }^{1}$ \\ Ricardo Pereira Reis ${ }^{2}$ \\ Luiz Carlos Takao Yamaguchi ${ }^{3}$
}

RESUMO: Por meio deste trabalho objetivou-se comparar duas metodologias de apuração de custos e identificar escalas na pecuária leiteira dos principais estados produtores de leite no Brasil. Estimaram-se as funções de custos de produção da atividade leiteira, considerando-se essas duas metodologias, definidas por "custo da atividade leiteira" e "centro de custos leite”. Analisaram-se dados técnicos e econômicos de 162 propriedades rurais, abrangendo o período de agosto de 2000 a julho de 2001 . A apuração de custos pela metodologia de "centro de custos" possibilitou uma maior precisão em relação às análises do custo total médio, evidenciando que, quando apurados os custos relacionados ao subsistema leite, os preços recebidos foram suficientes para remunerar o produtor. Ficou evidente a ocorrência de economias de escala. A análise dos indicadores evidencia que o estudo é composto por produtores que buscam maior eficiência produtiva e que ainda possuem possibilidades de ganho no que se refere à alocação e aproveitamento dos recursos. Entretanto, a possibilidade de redução dos custos médios é mais significativa para o sistema global de produção que para o subsistema leite, indicando que

\footnotetext{
${ }^{1}$ Mestre em Administração pela Universidade Federal de Lavras (UFLA) e Professor da Fundação de Ensino e Pesquisa do Sul de Minas (FEPESMIG) / UNIS - MG. pack.lopes@bol.com.br

${ }^{2}$ Professor Titular do Departamento de Administração e Economia (DAE) da UFLA.

${ }^{3}$ Doutor em Economia pela Universidade Federal de Viçosa (UFV) e Pesquisador da Empresa Brasileira de Pesquisa Agropecuária - Embrapa Gado de Leite.
} 
os demais subsistemas da atividade global apresentam um desempenho inferior quanto ao aproveitamento dos recursos produtivos e gerenciais em relação ao subsistema de produção de leite.

Palavras-chave: Pecuária leiteira, metodologia de custos, economias de escala.

Classificação JEL: Q13

Abstract: The present research had as objective, to compare checking cost methodologies and identify the milk production scales in the main states that produce milk in Brazil. The function of cost production of milk activity was estimated considering two methodologies, determined by: "milk activity cost" and "milk center cost". The technical and economic results of 162 producers were analyzed from August 2000 to July 2001. The cost investigation by the methodology of "milk center cost" made possible a bigger accuracy related to the analysis of the total medium cost in the milk production. It was clear that earnings occurrence with the production scale. The results make clear that the study is formed by producers that look for greater productive efficiency and that there is an even profit possibility when talking about better allocation and improvement of the resources. The indicators analysis pointed that the reduction possibility of medium cost is more significant for the production global system, that is, to the milk activity, than to the milk subsystem making clear that the other subsystems of the global activity presented a worse use of the resources than the milk production subsystem.

Key words: Cost methodology, dairy farming, center cost.

JEL Classification: Q13 - Agricultural Markets and Marketing; Cooperatives; Agribusiness.

\section{Introdução}

A cadeia produtiva do leite pode ser encontrada, mesmo que em diferentes aspectos, em todas as regiões brasileiras, atuando como uma atividade geradora de renda, tributos e empregos. 
As mudanças econômicas ocorridas na década de 1990 contribuíram como um divisor deste sistema agroindustrial, exigindo mudanças e ajustamentos estratégicos e estruturais do setor agroindustrial do leite (Reis et al., 2001). Nesse sentido, novos procedimentos têm sido adotados, visando ao aperfeiçoamento da gestão de custos e o incremento dos níveis de qualidade, desde a matéria-prima até o produto final.

Destaca-se que o setor produtivo, por representar o segmento mais vulnerável da cadeia, devido às limitações tecnológicas e gerenciais, é aquele que mais intensamente tem sofrido as conseqüências das novas exigências do mercado. Desta maneira, as relações que se estabelecem entre o setor agropecuário e os segmentos a montante e a jusante assumem, respectivamente, características de oligopólio e oligopsônio. Esta situação leva os produtores rurais a disporem de poucos recursos para negociarem seus interesses nos segmentos da cadeia produtiva do leite, inclusive à menor capacidade de negociação de preços (Alencar et al., 2001).

Diante deste cenário, caracterizado por declínio dos preços recebidos e limitado poder de negociação no mercado, por não conseguir controlar o preço do produto que vende, o produtor necessita administrar as variáveis que estão sob o seu controle. Sendo assim, de acordo com Fassio et al. (2005), uma das alternativas de que dispõem os produtores de leite para se manterem na atividade é a redução dos custos de produção, cujo conhecimento é essencial para o efetivo controle da empresa rural e para o processo de tomada de decisão.

Trata-se de uma estratégia para tornar seu produto competitivo. Dessa maneira, o resultado econômico em um mercado caracterizado pela estrutura de concorrência imperfeita dependerá do gerenciamento dos custos de produção e dos ganhos de escala. O aumento da eficiência produtiva torna-se fator decisivo para a competitividade do setor leiteiro (Reis et al., 2001).

Consideradas a grande heterogeneidade da cadeia produtiva do leite no Brasil e a sua presença em todo o território nacional, é salutar a identificação de indicadores técnicos e econômicos que possam permitir a utilização da gestão de custos como um instrumento de competitividade da pecuária leiteira nacional, facilitando uma compreensão mais clara das reais capacidades de resistência dos produtores nas diferentes realidades em que se encontram. A determinação das condições de efi- 
ciência econômica e dos níveis de escalas de produção na pecuária leiteira pode indicar a correta alocação dos recursos produtivos e auxiliar no estabelecimento de políticas públicas que considerem as diferenças regionais neste sistema produtivo.

Por meio deste estudo, buscou-se estimar os custos e as escalas de produção na pecuária leiteira dos estados de Minas Gerais, Goiás, São Paulo, Paraná e Rio Grande do Sul. Especificamente, os objetivos foram estimar e comparar os custos de produção da pecuária leiteira nos referidos estados, segundo duas metodologias, denominadas "custo da atividade leiteira" e "centro de custos leite", estimar as funções de custos e identificar a ocorrência de escalas de produção nos estados pesquisados, com base nestas metodologias.

\section{Metodologia}

\section{Referencial Teórico}

Um empresário, no processo produtivo, diante de ampla variedade de possíveis combinações, procura coordenar os fatores de produção de acordo com determinada tecnologia, com o objetivo de alcançar um certo nível de produção que proporcione a máxima eficiência econômica, ou seja, maximizar o lucro ou minimizar os custos.

Foi considerado, neste trabalho, que a empresa opera num mercado competitivo, não podendo, portanto, influenciar o preço de qualquer produto ou recurso utilizado no processo produtivo.

Ao se estudar o comportamento dos custos no longo prazo, considera-se período de tempo longo o bastante para que todos os recursos sejam passíveis de alterações. Embora a produção se dê no curto prazo, o longo prazo relaciona-se ao planejamento e às escolhas futuras de alternativas de produção, inclusive quanto ao tamanho do empreendimento. Assim, o conjunto das curvas de custo total médio no curto prazo (CTMeCP) determina a curva de custo médio no longo prazo (CMeLP), ou seja, a curva envelope ou a curva envoltória. A conformação da curva de CMeLP é de interesse na medida que fornece informações sobre o processo de produção em um período específico. Nas sucessivas situações de custos mínimos no curto prazo, um nível de produto, q, 
indica o tamanho de instalação ou escala de operação em que os custos médios mínimos em curto prazo e em longo prazo coincidem, ou seja, a escala ótima de produção. Este ponto se expressa pela igualdade:

$$
\mathrm{CMeLP}=\mathrm{CMaLP}=\mathrm{CTMeCP}=\mathrm{CMaCP} .
$$

O cálculo do custo total do leite, nesta pesquisa, foi realizado por meio de duas diferentes metodologias. A primeira metodologia refere-se ao modelo tradicional, denominado "custo da atividade leiteira", em que o custo de produção do leite é estimado a partir do custo total da atividade leiteira, deduzindo-se o valor dos animais descartados do custo total da atividade para se obter o custo do leite. A segunda metodologia se refere ao chamado "centro de custo leite", que foi analisado separadamente como um subsistema do sistema global da atividade leiteira, envolvendo apenas os custos incorridos especificamente para a produção de leite.

No modelo tradicional, primeira metodologia, o custo de produção é avaliado considerando a atividade leiteira como um todo. Como existe uma grande dificuldade em separar os recursos produtivos para a produção do leite e os que são alocados para os animais, o custo total de produção corresponde à atividade leiteira e não especificamente ao leite. Um dos motivos do freqüente uso desta metodologia refere-se ao procedimento para levantamento dos dados técnicos de produção e preços nas propriedades de leite, disponíveis, muitas das vezes, de forma bastante agregada.

Segundo Reis (2002), para apuração do custo específico do leite no modelo tradicional, o descarte de animais (bezerras e novilhas) entraria como receita da atividade leiteira e, a partir da dedução deste valor no custo total da atividade, ter-se-ia o custo de produção do leite. Outra alternativa seria considerar a venda destes animais como sub-produto da atividade. Neste estudo, optou-se pela primeira alternativa.

Os procedimentos utilizados nesta pesquisa para a estimativa dos custos de produção apurados pela metodologia do "custo da atividade leiteira” estão fundamentados na operacionalização dos recursos econômicos que compõem os custos fixos e variáveis, conforme operacionalização proposta pelo Centro Nacional de Pesquisa em Gado de Leite 
da Empresa Brasileira de Pesquisa Agropecuária (CNPGL/EMBRAPA). Os recursos analisados no processo produtivo da atividade leiteira foram: terra, benfeitorias, máquinas, equipamentos, rebanho produtivo (vacas, novilhas e bezerras), formação de pastagens, animais de tração e impostos fixos. Além do custo alternativo, estimou-se, com exceção do item terra, a depreciação de cada item. Quanto aos custos variáveis, consideraram-se as despesas com alimentação (silagem, farelo, ração, sal, milho, uréia etc.), produtos veterinários (antibiótico, bernicida, carrapaticida, vacina, vermífugo, material para inseminação etc.), mão-de-obra (permanente e temporária), serviços de terceiros, manutenção, combustível, energia elétrica, impostos variáveis e demais despesas gerais.

Para efeito de análise do custo alternativo dos recursos produtivos alocados no "custo da atividade leiteira", conforme operacionalização proposta pelo Centro Nacional de Pesquisa em Gado de Leite da Empresa Brasileira de Pesquisa Agropecuária, considerou-se a taxa de juros real de $6 \%$ a.a.. A exceção foi o item terra, cuja remuneração do capital investido foi de $3 \%$ a.a..

Em princípio, um sistema de produção pode ser considerado como uma incógnita, em que os insumos e os produtos são conhecidos e mensuráveis, embora o processo de transformação dos insumos em produtos seja desconhecido (Yamaguchi et al., 2002).

Entretanto, de acordo com Gastal (1980), uma análise realizada pelo processo de segmentação do sistema global permite desvendar alguns dos subsistemas que compõem o processo de produção, em que cada um corresponde a uma parte do processo de transformação. Nesse sentido, o segundo processo utilizado na estimativa dos custos nesta pesquisa refere-se à consideração metodológica levantada por autores como Tupy et al. (2000) e Yamaguchi (2000), que considera o uso da segmentação da produção de leite em subsistemas interligados entre si, aqui denominados de "centros de custos". Assim, este método leva em consideração subsistemas como, por exemplo, a produção de leite, a produção de animais para reposição, a produção de alimentos, dentre outros, conforme exemplifica a Figura 1. 
Figura 1. Centros de custos de produção de leite.

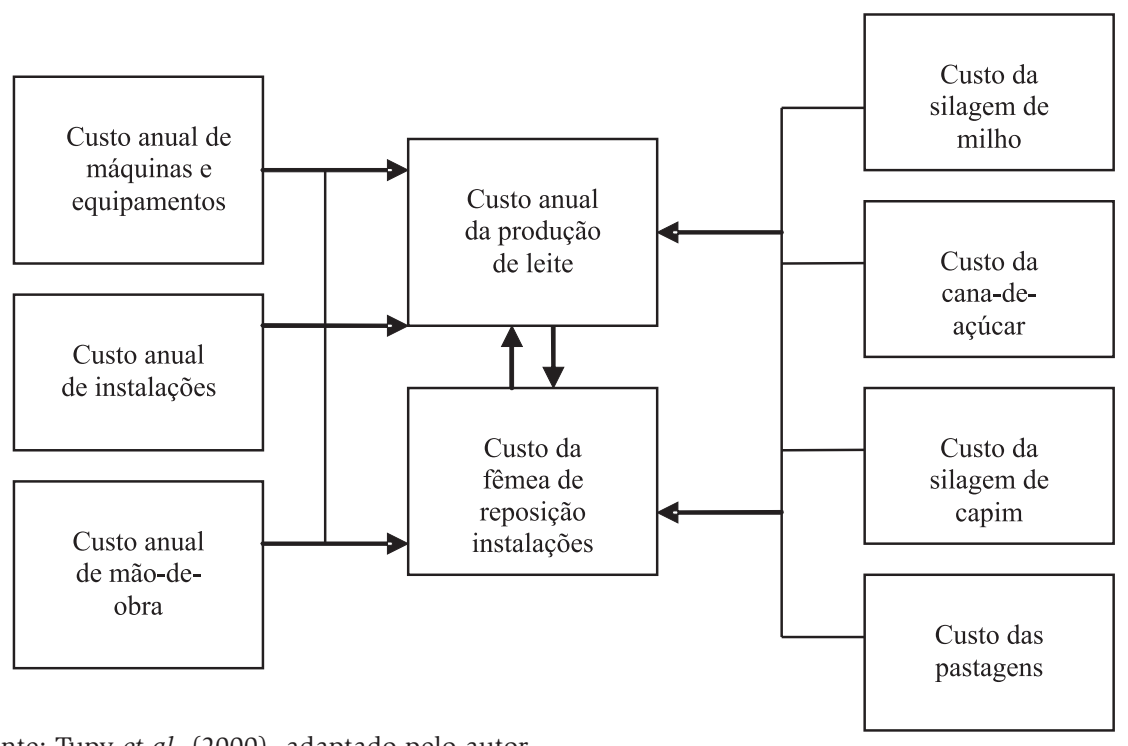

Fonte: Tupy et al. (2000), adaptado pelo autor.

A Figura 1, dessa maneira, representa sucintamente a estrutura na qual baseou-se e organizou-se o conjunto de planilhas para controle do custo de produção do leite elaboradas pelo CNPGL/EMBRAPA, que serviram de base para esta pesquisa. Nos subsistemas de produção de cana-de-açúcar, pastagens, silagens de milho e de capim são considerados os custos devido à manutenção, colheita e distribuição, benfeitorias e instalações, equipamentos, administração, consultorias e outras despesas, além de remuneração e depreciação. No subsistema de custo de mão-de-obra, incluem-se as despesas com ordenhador e com o trabalho de manejo (salário, $13^{\circ}$ salário e encargos sociais), bem como o salário do administrador. Nos subsistemas de custos de instalações, máquinas e equipamentos, têm-se os dispêndios relacionados às salas de ordenha e de leite, curral de espera e de manejo, rede hidráulica, reservatórios de água, tanque de resfriamento, balança de pesagem de animais, galpão e armazém, escritório, botijão de sêmen etc.

O subsistema de fêmeas de reposição possibilita estimar o custo total de manutenção anual do rebanho de fêmeas de reposição e o custo por novilha de reposição até o parto. Assim, neste subsistema, consideram- 
se as despesas com insumos, como leite para bezerro, concentrados, silagem, sal mineral, medicamentos, mão-de-obra, instalações, maquinário, despesas de manutenção, energia elétrica, combustíveis etc.

Finalmente, o subsistema utilizado para o custo de produção de leite leva em conta, como principais itens, as despesas de mão-de-obra, incluindo o trabalho familiar, despesas com alimentação, reprodução, produtos veterinários, dispêndios com instalações, máquinas e equipamentos, animais de produção, pastagens e despesas gerais.

Sendo assim, analisaram-se, nesta pesquisa, os custos de produção de leite apurados tanto pela metodologia tradicional, a partir dos "custos da atividade leiteira", quanto os apurados pela metodologia de "centros de custos", a partir da perspectiva da análise segmentada do sistema global de produção, conforme sugerido por Yamaguchi et al. (2001).

\section{Modelo analítico}

A base teórico-analítica deste estudo se fundamenta na teoria dos custos de produção. A escolha da forma funcional deve considerar, além da capacidade desta para a constatação de economia de escala, as propriedades inerentes ao comportamento dos custos.

Considerando-se que o custo total (CT) para se produzir e negociar determinado número de unidades de um produto é uma função somente de q, então, a função de custo total pode ser representada por CT $=\mathrm{f}(\mathrm{q})$. Nesse sentido, a partir da função do custo total, outros custos podem ser derivados. Assim, podem ser obtidos o custo variável médio, o custo total médio e o custo marginal.

As formas funcionais quadrática, cúbica e potência foram testadas para representar o comportamento do custo total. A escolha da forma apropriada buscou verificar a hipótese de formato em U para a curva de custo médio por meio dos modelos quadrático e cúbico, ou, se não, com o modelo potência.

Para que a função quadrática seja coerente com a teoria do custo, o coeficiente do termo quadrático $\left(\beta_{2}\right)$ deve ser positivo. Neste caso, o custo marginal cresce com a produção e o custo médio apresenta a forma em U: 


$$
C T=\beta_{2} q^{2}+\beta_{1} q+\beta_{0}+\varepsilon,
$$

em que CT é o custo total de produção (R\$), q a produção anual (litros), $\beta$ 's os coeficientes estimados e $\varepsilon$ é o termo do erro que se admite possuir as propriedades usuais.

$\mathrm{Na}$ alternativa cúbica, espera-se do coeficiente do termo cúbico $\left(\beta_{3}\right)$ sinal positivo e do coeficiente do termo quadrático $\left(\beta_{2}\right)$ valor nulo ou negativo:

$$
C T=\beta_{3} q^{3}+\beta_{2} q^{2}+\beta_{1} q+\beta_{0}+\varepsilon,
$$

em que $\varepsilon$ é o termo do erro que se admite possuir as propriedades usuais, os $\beta$ 's são os coeficientes a serem estimados, q a produção anual (litros) e CT o custo total de produção (R\$).

Para o modelo de função potência, os coeficientes $\beta_{0}$ e $\beta_{1}$ devem possuir sinais positivos. Entretanto, situações podem se configurar conforme a estimativa do coeficiente $\beta_{1}$ :

$$
\mathrm{CT}=\beta_{0} \mathrm{q}^{\beta 1} .
$$

Se a estimativa do coeficiente $\beta_{1}$ for maior que a unidade, o custo total aumenta a taxas crescentes com o crescimento da produção, sendo também crescente o custo marginal, enquanto o custo médio decresce. Sendo o coeficiente $\beta_{1}$ menor que um e maior que zero, o custo total cresce a taxas decrescentes com o aumento da produção, enquanto o custo marginal e o custo médio decrescem continuamente, sem apresentar um ponto mínimo.

O coeficiente $\beta_{1}$ representa a elasticidade custo (Ec) em relação à produção, isto é, a variação proporcional no custo resultante de uma variação proporcional na produção.

Tendo por referência a elasticidade custo da produção, pode ser auferido o tipo de retorno à escala (RE), uma vez que este é o inverso da elasticidade custo, bem como a ocorrência de economia ou deseconomia de escala (EE).

Dado que:

$$
\mathrm{RE}=1 / \mathrm{Ec},
$$


então, se RE for maior, igual ou menor que a unidade, têm-se retornos crescentes, constantes ou decrescentes à escala.

Dado que:

$\mathrm{EE}=1-\mathrm{Ec}$,

então, o comportamento da empresa em relação ao nível de operação é economicamente eficaz quando EE é positivo, ou seja, estão ocorrendo economias de escala nos níveis operados. Se EE é negativo, há deseconomias de escala.

Foi utilizado o método dos mínimos quadrados ordinários (MQO) para ajustar os modelos econométricos propostos. A avaliação da qualidade do ajustamento dos dados foi efetuada por meio dos coeficientes de determinação $\left(R^{2}\right)$ e de determinação ajustado $\left(R^{2} A j\right.$.), os quais auferem a proporção da alteração na variável dependente estimada, que é explicada pelo comportamento da variável independente. Também a estatística $\mathrm{F}$ foi empregada para avaliar estatisticamente a significância da relação entre as variáveis custo total e quantidade produzida. Na avaliação dos coeficientes estimados, além da coerência dos sinais, o teste $t$ mediu a significância destes para o modelo.

\section{Região do estudo e dados básicos}

Os dados utilizados neste estudo foram cedidos pelo Centro Nacional de Pesquisa em Gado de Leite da Empresa Brasileira de Pesquisa Agropecuária (CNPGL/EMBRAPA); eles foram coletados em junho de 2002 e referem-se ao período de agosto de 2000 a julho de 2001 . São derivados do processamento de informações de natureza estrutural, tecnológica e econômica de 162 propriedades rurais localizadas nos principais estados produtores de leite do país, ou sejam, os estados de Minas Gerais, Goiás, São Paulo, Paraná e Rio Grande do Sul.

As variáveis empregadas nos modelos foram o custo total, expresso em reais, apropriado tanto pelo processo tradicional (custo da atividade leiteira), quanto pelo processo de segmentação do sistema global de produção de leite (centro de custo leite), e a quantidade produzida, expressa em litros de leite por ano. 
O custo alternativo teve como referência a taxa de juro real de $6 \%$ ao ano. A remuneração anual do capital investido em terra foi de $3 \%$, tomando-se como base o valor de venda do hectare na região em que se localizava a propriedade. Para o fator benfeitorias e os demais fatores, foi estipulada a taxa de $6 \%$ a.a. sobre o valor médio. A composição do grupo de produtores observados resultou da seleção intencional destes em função da disponibilidade de dados que atendessem aos objetivos propostos.

Em consonância com estes objetivos, optou-se pela caracterização dos estratos com base nos estados onde estão localizados os produtores. Essa caracterização também se mostrou mais adequada devido ao fato de, nas observações que compõem o estudo, predominarem fortemente as características tecnológicas e econômicas relacionadas aos grandes produtores, desaconselhando qualquer caracterização dos estratos por níveis de produção diária de leite.

Este fato pode ser observado na Tabela 1, que demonstra as freqüências relativas do número de produtores de leite estudados por estrato de produção diária e a distribuição percentual da produção. Considerou-se, para efeitos de comparação, a definição dos sistemas de produção utilizada pelo SEBRAE-FAEMG (1996).

Tabela 1. Freqüência relativa do número de produtores de leite estudados, por estrato de produção diária e distribuição percentual da produção, agosto de 2000 a julho de 2001.

\begin{tabular}{lccc}
\hline \multicolumn{1}{c}{ Estrato de produção } & $\mathbf{N}^{\mathbf{0}}$ de produtores & \% Total da amostra & \% Produção \\
\hline Até $\mathbf{5 0}$ I/dia & 0 & 0 & 0 \\
De $\mathbf{5 1}$ a 250 I/dia & 21 & 12,96 & 3,57 \\
Acima de 250 l/dia & 141 & 87,04 & 96,43 \\
\hline Total & 162 & 100 & 100 \\
\hline
\end{tabular}

Fonte: Dados da pesquisa.

A caracterização dos estratos também se baseou em indicadores econômicos e de produtividade, tendo sido testadas estatisticamente as diferenças entre os estados estudados. Para tanto, efetuou-se a análise de variância dos indicadores considerados, utilizando-se o sistema computacional Statistics Package for the Social Science (SPSS for Windows). Se a análise de variância rejeitou a hipótese nula, pelo menos a 
$5 \%$ de probabilidade, testou-se a hipótese de igualdade das médias por meio do teste de Tukey, a $5 \%$ de probabilidade.

Para cada variável, além das informações sobre a média, também foram apresentados os coeficientes de variação, para que se pudesse visualizar o grau de heterogeneidade nos estratos de produção e em todo o grupo de produtores analisados.

\section{Resultados e Discussão}

\section{Resultados econômicos dos produtores de leite em estudo}

São apresentadas nesta seção as estimativas de alguns parâmetros que possibilitam sintetizar o diagnóstico econômico da produção leiteira nos estados em estudo.

Observa-se, na Tabela 2, que, considerando-se os "custos totais médios da atividade leiteira", apurados pelo sistema tradicional, foram observadas médias de $\mathrm{R} \$$ 0,31/litro para o Estado de Goiás, R \$ 0,33/litro para Minas Gerais, $\mathrm{R} \$ 0,31 /$ litro para o Paraná, $\mathrm{R} \$ 0,34 /$ litro para o Rio Grande do Sul e R\$ 0,40/litro para São Paulo. As médias para os custos totais médios do "centro de custo leite", por estado, foram de R $\$$ 0,28/litro para o Estado de Goiás, R\$ 0,30/litro para Minas Gerais, R\$ 0,27/litro para o Paraná, R \$ ,27/litro para o Estado do Rio Grande do Sul e R\$ 0,34/litro para São Paulo.

Tabela 2. Custo total médio, por estado produtor, apurado pelas metodologias "custo da atividade leiteira" e "centro de custo leite", em R \$/litro, agosto de 2000 a julho de 2001.

\begin{tabular}{lcccc}
\hline & \multicolumn{4}{c}{ Métodos de apuração dos custos } \\
\cline { 2 - 5 } Estado & Atividade leiteira & $\mathbf{C V}^{\mathbf{1}} \mathbf{( \% )}$ & Centro de custo leite & $\mathbf{C V}^{\mathbf{1}} \mathbf{( \% )}$ \\
\hline Goiás & $0,31 \mathrm{a}$ & 25,44 & $0,28 \mathrm{a}$ & 19,56 \\
Minas Gerais & $0,33 \mathrm{a}$ & 21,69 & $0,30 \mathrm{a}$ & 16,52 \\
Paraná & $0,31 \mathrm{a}$ & 19,10 & $0,27 \mathrm{a}$ & 16,22 \\
Rio Grande do Sul & $0,34 \mathrm{ab}$ & 19,79 & $0,27 \mathrm{a}$ & 15,36 \\
São Paulo & $0,40 \mathrm{~b}$ & 33,08 & $0,34 \mathrm{~b}$ & 18,77 \\
\hline GERAL & 0,34 & 26,39 & 0,29 & 19,54 \\
\hline
\end{tabular}

Fonte: Dados da pesquisa.

Médias seguidas da mesma letra, na coluna, são estatisticamente iguais pelo teste de Tukey, a 5\% de probabilidade.

${ }^{1}$ Coeficiente de variação. 
Considerando-se o que foi apurado pela metodologia do "custo da atividade leiteira", sistema tradicional, observa-se que o custo total médio para o Estado de São Paulo foi significativamente superior ao dos demais estados. O Estado do Rio Grande do Sul também apresentou um custo significativamente superior ao dos estados de Goiás, Paraná e Minas Gerais, sendo menor apenas que o do Estado de São Paulo.

Entretanto, os custos totais médios do subsistema leite para os estados estudados, apurados pela metodologia do "centro de custo leite", apresentaram-se significativamente iguais entre si, com exceção novamente do Estado de São Paulo, que apresentou um custo significativamente superior ao dos demais.

A análise dos dados da Tabela 2 também demonstra que a apuração pela metodologia do "centro de custo leite" permite uma melhor avaliação sobre a existência ou não de diferenças estatisticamente significativas entre os custos nos estados. Quando avaliado pela metodologia do "custo da atividade leiteira", o Estado do Rio Grande do Sul apresentou-se significativamente diferente dos demais. Entretanto, quando apurado pela metodologia do "centro de custo leite", tal diferença não se confirmou, proporcionando uma avaliação mais precisa e específica em relação aos custos de produção do leite nos estados. Esta maior precisão permite visualizar que o subsistema de produção de leite do Estado do Rio Grande do Sul é tão eficiente quanto o dos demais, estando sua menor eficiência, provavelmente, em outros subsistemas de produção da atividade leiteira.

Ainda em relação à Tabela 2, verifica-se que entre o custo apurado pela metodologia do custo total da atividade e o custo apurado para o centro de custo leite, existe uma diferença de $11 \%$ para o Estado de Goiás, $10 \%$ para Minas Gerais, $15 \%$ para o Paraná, 26\% para o Rio Grande do Sul e $18 \%$ para São Paulo. De maneira global, o custo total apurado pela metodologia tradicional foi $17 \%$ superior ao custo apurado para o subsistema leite.

O volume de produção é um indicador muito importante por exercer grande influência sobre o comportamento dos custos de produção. Os dados da Tabela 3 mostram a produção média diária e a produção média anual dos referidos estados produtores. 
Tabela 3. Produção média diária e anual das propriedades estudadas, por estado produtor, agosto de 2000 a julho de 2001.

\begin{tabular}{lcccc}
\hline & \multicolumn{2}{c}{ Produção diária } & \multicolumn{2}{c}{ Produção anual } \\
\cline { 2 - 5 } Estado & Média (1/dia) & $\mathbf{C V}^{\mathbf{1}} \mathbf{( \% )}$ & Médias (l/ano) & $\mathbf{C V}^{\mathbf{1}} \mathbf{( \% )}$ \\
\hline Goiás & $552 \mathrm{a}$ & 49,06 & $201494 \mathrm{a}$ & 49,06 \\
Minas Gerais & $823 \mathrm{ab}$ & 45,81 & $300322 \mathrm{ab}$ & 45,81 \\
Paraná & $1051 \mathrm{~b}$ & 108,18 & $383553 \mathrm{~b}$ & 108,18 \\
Rio Grande do Sul & $694 \mathrm{ab}$ & 84,78 & $253225 \mathrm{ab}$ & 84,78 \\
São Paulo & $572 \mathrm{a}$ & 64,42 & $208694 \mathrm{a}$ & 64,42 \\
\hline GERAL & 735 & 87,74 & 268255 & 87,74 \\
\hline
\end{tabular}

Fonte: Dados da pesquisa.

Médias seguidas da mesma letra, na coluna, são estatisticamente iguais pelo teste de Tukey, a $5 \%$ de probabilidade.

${ }^{1}$ Coeficiente de variação.

O Estado do Paraná apresentou o maior nível de produção, sendo $90 \%$ e $84 \%$, respectivamente, superior ao dos estados de Goiás e de São Paulo, que apresentaram a menor média diária. Os estados de Minas Gerais e Rio Grande do Sul apresentaram níveis de produção intermediários entre os estados estudados.

A obtenção de maiores níveis de produtividade é condição necessária para um desempenho econômico eficiente. Nesse sentido, considerou-se, neste trabalho, a produtividade média diária por vaca em lactação. Os dados da Tabela 4 indicam que, considerando-se 300 dias de lactação por vaca/ano, conforme metodologia utilizada por SEBRAEFAEMG (1996), a presente pesquisa encontrou uma produtividade média de 9,86 litros/vaca em lactação/dia para o Estado de Goiás, 12,32 para Minas Gerais, 18,91 para o Paraná, 18,77 para o Rio Grande do Sul e 14,41 para São Paulo. A produtividade média global foi de 15,14 litros/vaca em lactação/dia. A produtividade média diária foi obtida por meio do ajuste dos dados da pesquisa, transformando a produção anual para produção por período de lactação de 10 meses.

Os estados do Paraná e do Rio Grande do Sul apresentaram melhor desempenho em relação à produtividade, seguidos pelo Estado de São Paulo. O pior desempenho foi apresentado pelo Estado de Goiás. Minas Gerais apresentou um desempenho superior apenas ao do Estado de Goiás.

Analisando-se os dados das Tabelas 5 e 6, relacionam-se as produtividades dos rebanhos e os volumes de produção. Com exceção dos estados de Minas Gerais e São Paulo, as maiores produtividades acompanharam o 
aumento do nível de produção, demonstrando que maiores produtividades em relação a vacas em lactação são cruciais para se obterem escalas de produção. Percebe-se também a relação entre os níveis de produção, a produtividade e os custos estimados nas propriedades, seja considerando a relação custo fixo sobre o custo total ou os custos totais médios da produção de leite. Os dados da Tabela 5 demonstram os custos apurados pela metodologia do "custo da atividade leiteira" e os da Tabela 6 apresentam os custos apurados pela metodologia de "centro de custo leite".

Tabela 4. Produtividade média diária por vaca em lactação, por estado produtor, agosto de 2000 a julho de 2001.

\begin{tabular}{lcc}
\hline & \multicolumn{2}{c}{ Produtividade por vaca em lactação } \\
\cline { 2 - 3 } Estado & (l/vaca em lactação/dia) & $\mathbf{C V}^{\mathbf{1}} \mathbf{( \% )}$ \\
\hline Goiás & $9,86 \mathrm{a}$ & 29,59 \\
Minas Gerais & $12,32 \mathrm{ab}$ & 25,81 \\
Paraná & $18,91 \mathrm{c}$ & 26,92 \\
Rio Grande do Sul & $18,77 \mathrm{c}$ & 24,94 \\
São Paulo & $14,41 \mathrm{~b}$ & 43,23 \\
\hline GERAL & 15,14 & 38,21 \\
\hline
\end{tabular}

Fonte: Dados da pesquisa.

Médias seguidas da mesma letra, na coluna, são estatisticamente iguais pelo teste de Tukey, a $5 \%$ de probabilidade.

${ }^{1}$ Coeficiente de variação.

Tabela 5. Volume médio de produção, produtividade, percentual do custo fixo sobre o custo total e custo total médio, por estado produtor, para a metodologia "custo da atividade leiteira", agosto de 2000 a julho de 2001.

\begin{tabular}{lccccc}
\hline & Produtividade & & \multicolumn{3}{c}{ Custo da atividade leiteira } \\
\cline { 4 - 6 } \multicolumn{1}{c}{ Estado } & (1/vacas lactação/dia) & Volume (l/dia) & CFT/CT (\%) & CV $^{\mathbf{1}} \mathbf{( \% )}$ & CTMe (RS/l) \\
\hline Goiás & 9,86 & 552 & $32,80 \mathrm{c}$ & 23,81 & 0,31 \\
Minas Gerais & 12,32 & 823 & $27,52 \mathrm{ab}$ & 26,85 & 0,33 \\
Paraná & 18,91 & 1051 & $24,73 \mathrm{a}$ & 22,46 & 0,31 \\
Rio Grande do Sul & 18,77 & 694 & $25,26 \mathrm{a}$ & 28,90 & 0,34 \\
São Paulo & 14,41 & 572 & $31,86 \mathrm{bc}$ & 28,10 & 0,40 \\
\hline GERAL & 15,14 & 735 & 28,43 & 28,68 & 0,34 \\
\hline
\end{tabular}

Fonte: Dados da pesquisa.

Médias seguidas da mesma letra, na coluna, são estatisticamente iguais pelo teste de Tukey, a $5 \%$ de probabilidade.

${ }^{1}$ Coeficiente de variação.

Segundo Marques et al. (2002), produtores com maiores escalas de produção apresentam uma relação CFT/CT expressivamente mais bai- 
xa. Analisando-se os dados das Tabelas 4 e 5, observa-se que os estados com maior volume de produção realmente demonstraram esta redução quando comparados aos estados com menor volume de produção.

Tabela 6. Volume médio de produção, produtividade, percentual do custo fixo sobre o custo total e custo total médio, por estado produtor, para a metodologia "centro de custo leite", agosto de 2000 a julho de 2001.

\begin{tabular}{lccccc}
\hline & \multicolumn{2}{c}{$\begin{array}{c}\text { Produtividade } \\
\text { Estado }\end{array}$} & & \multicolumn{3}{c}{ Centro custo leite } \\
\cline { 4 - 6 } & (1/vacas lactação/dia) & Volume (l/dia) & CFT/CT (\%) & CV $^{\mathbf{1}}$ (\%) & CTMe (RS/l) \\
\hline Goiás & 9,86 & 552 & $16,99 \mathrm{c}$ & 25,55 & 0,28 \\
Minas Gerais & 12,32 & 823 & $13,51 \mathrm{a}$ & 24,18 & 0,30 \\
Paraná & 18,91 & 1051 & $15,59 \mathrm{ab}$ & 30,29 & 0,27 \\
Rio Grande do Sul & 18,77 & 694 & $15,81 \mathrm{ab}$ & 29,58 & 0,27 \\
São Paulo & 14,41 & 572 & $17,18 \mathrm{c}$ & 31,55 & 0,34 \\
\hline GERAL & 15,14 & 735 & 15,82 & 29,52 & 0,29 \\
\hline
\end{tabular}

Fonte: Dados da pesquisa.

Médias seguidas da mesma letra, na coluna, são estatisticamente iguais pelo teste de Tukey, a $5 \%$ de probabilidade.

${ }^{1}$ Coeficiente de variação.

Ainda em relação aos dados das Tabelas 5 e 6, percebe-se também que a relação CFT/CT é menor quando apurados somente os custos relacionados exclusivamente ao "centro de custo leite" do que quando apurados os custos pela metodologia tradicional. Constata-se, desse modo, que a produção de leite, especificamente, possui custos variáveis em uma proporção muito maior do que quando se considera a atividade leiteira como um todo, demonstrando uma maior especialização do subsistema leite.

Na Tabela 7 tem-se o desempenho econômico da produção leiteira nos estados pesquisados, especificando-se os valores médios dos custos de produção unitários do leite, bem como o resultado do preço médio recebido por unidade de produção.

A análise da Tabela 7 demonstra que, considerando-se a apuração pela metodologia "custo da atividade leiteira", tem-se que o custo variável médio é maior para os estados do Rio Grande do Sul e de São Paulo. Entretanto, quando se considera a apuração pela metodologia "centro de custo leite”, tem-se que o custo variável médio é maior nos estados de São Paulo e Minas Gerais.

Os dados da Tabela 7 também demonstram que os produtores dos estados de São Paulo e de Minas Gerais apresentaram um preço médio recebi- 
do superior ao dos produtores dos demais estados. O preço médio recebido pelo leite de maneira geral foi de $\mathrm{R} \$ 0,35$ por litro. Percebe-se que os custos variáveis médios são cobertos pelo preço médio recebido em todos os estados, tanto para a metodologia "custo da atividade leiteira" quanto para a metodologia "centro de custo leite". Isso indica que a pecuária leiteira nos estados estudados tem condições de continuar a produzir no curto prazo.

Tabela 7. Desempenho econômico dos estados produtores de leite pesquisados, agosto de 2000 a julho de 2001.

\begin{tabular}{|c|c|c|c|c|c|c|}
\hline \multirow[b]{2}{*}{ Receitas e Custos (R\$/l) } & \multicolumn{6}{|c|}{ Estados produtores } \\
\hline & GO & MG & PR & RS & SP & GERAL \\
\hline & \multicolumn{6}{|c|}{ Custo da atividade leiteira } \\
\hline CVMe & $0,18 \mathrm{a}$ & $0,22 a b$ & $0,21 \mathrm{ab}$ & $0,23 \mathrm{~b}$ & $0,25 \mathrm{~b}$ & 0,22 \\
\hline$C V^{1}(\%)$ & 30,87 & 25,19 & 22,65 & 23,84 & 39,10 & 30,36 \\
\hline \multirow[t]{2}{*}{ CTMe } & 0,31 & 0,33 & 0,31 & 0,34 & 0,40 & 0,34 \\
\hline & \multicolumn{6}{|c|}{ Centro de custo leite } \\
\hline CVMe & $0,21 \mathrm{a}$ & $0,24 \mathrm{~b}$ & $0,21 \mathrm{a}$ & $0,20 \mathrm{a}$ & $0,26 \mathrm{~b}$ & 0,22 \\
\hline$C V^{1}(\%)$ & 20,92 & 17,58 & 18,66 & 17,72 & 17,45 & 20,62 \\
\hline CTMe & 0,28 & 0,30 & 0,27 & 0,27 & 0,34 & 0,29 \\
\hline Preço Médio Recebido & $0,33 \mathrm{a}$ & $0,39 \mathrm{~b}$ & $0,34 \mathrm{a}$ & $0,33 \mathrm{a}$ & $0,37 \mathrm{~b}$ & 0,35 \\
\hline $\mathrm{CV}^{1}(\%)$ & 9,73 & 5,59 & 7,05 & 7,43 & 6,77 & 10,01 \\
\hline
\end{tabular}

Fonte: Dados da pesquisa.

Médias seguidas da mesma letra, na linha, são estatisticamente iguais pelo teste de Tukey, a $5 \%$ de probabilidade.

${ }^{1}$ Coeficiente de variação.

Observando-se os dados da Tabela 8, identifica-se que os custos apurados pela metodologia "custo da atividade leiteira" têm os piores desempenhos econômicos nos estados de São Paulo e Rio Grande do Sul. Neste estados, portanto, parte da depreciação do capital fixo não está sendo reposta; a persistir tal situação, os pecuaristas destes estados perdem sua capacidade produtiva e, no longo prazo, poderão buscar outras alternativas de aplicação do capital. Entretanto, ao considerar-se o custo apurado para o "centro de custo leite", percebe-se que os preços recebidos pelos produtores foram suficientes para cobrir os seus custos totais médios, possibilitando-lhes, no curto prazo, a permanência na atividade. No longo prazo, entretanto, tal situação estimulará a entrada de novos produtores no mercado. Os dados da Tabela 8 também evidenciam que o Estado de Minas Gerais apresentou um resultado significativamente melhor em relação aos demais estados estudados. 
Tabela 8. Resultado econômico dos estados produtores de leite pesquisados, agosto de 2000 a julho de 2001.

\begin{tabular}{|c|c|c|c|c|c|c|}
\hline \multirow[b]{2}{*}{ Resultado econômico } & \multicolumn{6}{|c|}{ Estados produtores } \\
\hline & GO & MG & PR & RS & SP & GERAL \\
\hline & \multicolumn{6}{|c|}{ Custo da atividade leiteira } \\
\hline CTMe (R\$/l) & 0,31 & 0,33 & 0,31 & 0,34 & 0,40 & 0,34 \\
\hline Preço médio recebido (R\$/I) & 0,33 & 0,39 & 0,34 & 0,33 & 0,37 & 0,35 \\
\hline \multirow[t]{2}{*}{ Resultado (R\$/I) } & 0,02 ab & $0,06 \mathrm{c}$ & 0,03 ab & $-0,01 a$ & $-0,03 a$ & $\overline{0,01}$ \\
\hline & \multicolumn{6}{|c|}{ Centro de custo leite } \\
\hline CTMe (R\$/I) & 0,28 & 0,30 & 0,27 & 0,27 & 0,34 & 0,29 \\
\hline Preço médio recebido (R\$/I) & 0,33 & 0,39 & 0,34 & 0,33 & 0,37 & 0,35 \\
\hline Resultado (R\$/I) & 0,05 ab & $0,09 \mathrm{c}$ & 0,07 bc & 0,06 abc & 0,03 a & $\overline{0,06}$ \\
\hline
\end{tabular}

Fonte: Dados da pesquisa.

Médias seguidas da mesma letra, na linha, são estatisticamente iguais pelo teste de Tukey, a $5 \%$ de probabilidade.

\section{A função de custo total}

Para verificação de ocorrências de economias de escala, a função na forma de potência foi selecionada por atender tanto à coerência dos sinais quanto à significância estatística das estimativas e da regressão. Os resultados do ajustamento estatístico do custo total e quantidade produzida para os estados estudados, tanto individualmente quanto de maneira geral, considerando-se as duas metodologias de apuração de custos, estão sintetizados na Tabela 9.

Tabela 9. Estimativas de função de "custo para a atividade leiteira" e para o "centro de custo leite", nos estados pesquisados, agosto de 2000 a julho de 2001 .

\begin{tabular}{|c|c|c|c|c|c|c|}
\hline \multirow[b]{2}{*}{ Estados } & \multicolumn{6}{|c|}{ Parâmetros } \\
\hline & 0 & 1 & $t *$ & $\overline{\mathbf{R}^{2}}$ & $\overline{R^{2} \mathbf{A j}}$ & $F^{*}$ \\
\hline & \multicolumn{6}{|c|}{ Custo da atividade leiteira } \\
\hline Goiás & 2,132520 & 0,838237 & 8,3300 & 0,7126 & 0,7023 & 69,4100 \\
\hline Minas Gerais & 0,436538 & 0,974893 & 9,5080 & 0,7635 & 0,7551 & 90,3984 \\
\hline Paraná & 0,814867 & 0,920352 & 23,7990 & 0,9529 & 0,9512 & 566,4129 \\
\hline Rio Grande do Sul & 1,140416 & 0,899158 & 22,8990 & 0,9291 & 0,9274 & 524,3737 \\
\hline São Paulo & 0,628408 & 0,955985 & 8,0920 & 0,7005 & 0,6898 & 65,4727 \\
\hline \multirow[t]{2}{*}{ GERAL } & 1,013164 & 0,907200 & 28,9540 & 0,8397 & 0,8387 & 838,3278 \\
\hline & \multicolumn{6}{|c|}{ Centro de custos leite } \\
\hline Goiás & 1,983123 & 0,837317 & 12,4710 & 0,8474 & 0,8420 & 155,5339 \\
\hline Minas Gerais & 0,329197 & 0,990838 & 12,9260 & 0,8565 & 0,8513 & 167,0772 \\
\hline Paraná & 0,489584 & 0,950354 & 29,7290 & 0,9693 & 0,9682 & 883,8221 \\
\hline Rio Grande do Sul & 0,435580 & 0,960220 & 28,1580 & 0,9520 & 0,9508 & 792,8682 \\
\hline São Paulo & 0,474377 & 0,971441 & 18,1540 & 0,9217 & 0,9189 & 329,5828 \\
\hline GERAL & 0,591924 & 0,940425 & 43,3670 & 0,9216 & 0,9211 & 1880,6991 \\
\hline
\end{tabular}

Fonte: Dados da pesquisa.

* Nível de significância de $5 \%$. 
Segundo o teste $\mathrm{t}$ aplicado, os coeficientes estimados apresentaram os sinais esperados e de significância de $5 \%$. Os valores observados para a estatística "F" indicam que a regressão foi altamente significativa.

De acordo com os coeficientes de determinação ajustados $\left(R^{2} A j.\right)$, observa-se que a estimativa do custo total foi influenciada pela especificação do volume de produção com diferentes intensidades entre os estados estudados.

De maneira geral, a produção de leite influenciou em 83,87\% e $92,11 \%$ as variações nas estimativas do custo total, considerando-se as apurações pelas metodologias tradicional e de centro de custos, respectivamente.

As elasticidades de custo total, representadas pelos $\beta_{1}$ 's, apresentaram-se menores que a unidade, indicando taxas decrescentes de crescimento do custo total com a quantidade produzida, ou seja, os gastos com fatores de produção resultaram em maior volume de produção.

Dessa maneira, pode-se afirmar que variações proporcionais na produção são alcançadas com variações menos que proporcionais nos insumos utilizados.

Para todos os estados estudados, o custo total variou menos que proporcionalmente em relação à produção, indicando taxas de crescimento decrescentes do custo total em função do aumento no volume produzido, o que corresponde a taxas de rendimentos crescentes à escala de produção.

\section{As funções de custo total médio e custo marginal}

Partindo das funções ajustadas para o custo total foram obtidas as equações do custo total médio para os estados estudados. De acordo com as características das funções de custo médio e custo marginal, observa-se que decrescem à medida que o volume de produção aumenta, apresentando tendência única de decrescimento, o que também evidencia economias de escala. As funções de custo médio e marginal obtidos, considerando a metodologia de apuração pelo "custo da atividade leiteira", por estado e geral, foram as seguintes: 


$$
\begin{aligned}
& \mathrm{CTMe}_{\mathrm{GO}}=2,132520 \mathrm{q}^{-0,161763} \\
& \mathrm{CTMe}_{\mathrm{MG}}=0,436538 \mathrm{q}^{-0,025107} \\
& \mathrm{CTMe}_{\mathrm{PR}}=0,814867 \mathrm{q}^{-0,079648} \\
& \mathrm{CTMe}_{\mathrm{RS}}=1,140416 \mathrm{q}^{-0,100842} \\
& \mathrm{CTMe}_{\mathrm{SP}}=0,628408 \mathrm{q}^{-0,044015} \\
& \mathrm{CTMe}_{\text {GERAL }}=1,013164 \mathrm{q}^{-0,0928}
\end{aligned}
$$$$
\mathrm{CMa}_{\mathrm{GO}}=1,787557 \mathrm{q}^{-0,161763}
$$$$
\mathrm{CMa}_{\mathrm{MG}}=0,425578 \mathrm{q}^{-0,025107}
$$$$
\mathrm{CMa}_{\mathrm{PR}}=0,749964 \mathrm{q}^{-0,079648}
$$$$
\mathrm{CMa}_{\mathrm{RS}}=1,025414 \mathrm{q}^{-0,100842}
$$$$
\mathrm{CMa}_{\mathrm{SP}}=0,600749 \mathrm{q}^{-0,044015}
$$$$
\mathrm{CMa}_{\text {GERAL }}=0,919142 \mathrm{q}^{-0,0928}
$$

Considerando-se a metodologia de apuração pela metodologia "centro de custos leite" como um todo, corresponderam às funções de custo médio e marginal, por estado e geral, as seguintes expressões:

$$
\begin{aligned}
& \mathrm{CTMe}_{\mathrm{GO}}=1,983123 \mathrm{q}^{-0,162683} \\
& \mathrm{CTMe}_{\mathrm{MG}}=0,329197 \mathrm{q}^{-0,009162} \\
& \mathrm{CTMe}_{\mathrm{PR}}=0,489584 \mathrm{q}^{-0,049646} \\
& \mathrm{CTMe}_{\mathrm{RS}}=0,435580 \mathrm{q}^{-0,03978} \\
& \mathrm{CTMe}_{\mathrm{SP}}=0,474377 \mathrm{q}^{-0,028559} \\
& \mathrm{CTMe}_{\text {GERAL }}=0,591924 \mathrm{q}^{-0,059575}
\end{aligned}
$$

$$
\begin{aligned}
& \mathrm{CMa}_{\mathrm{GO}}=1,660503 \mathrm{q}^{-0,162683} \\
& \mathrm{CMa}_{\mathrm{MG}}=0,326181 \mathrm{q}^{-0,009162} \\
& \mathrm{CMa}_{\mathrm{PR}}=0,465278 \mathrm{q}^{-0,049646} \\
& \mathrm{CMa}_{\mathrm{RS}}=0,418253 \mathrm{q}^{-0,03978} \\
& \mathrm{CMa}_{\mathrm{SP}}=0,460829 \mathrm{q}^{-0,028559} \\
& \mathrm{CMa}_{\text {GERAL }}=0,556660 \mathrm{q}^{-0,059575}
\end{aligned}
$$

Os sinais negativos dos expoentes expressam o comportamento decrescente dos custos médios à medida que o volume de produção aumenta. Embora não identifique um ponto de menor custo, sua inclinação negativa indica que as propriedades analisadas estão tendo ganhos de escala, isto é, os custos unitários decrescem com o aumento da produção.

Esta conformação das curvas de custos médios indica que volumes maiores de produção são significativos para empresas de produção de leite, no sentido de levarem à maior eficiência econômica. Além de maiores níveis de produção proporcionarem ganhos de escala, este formato das curvas de custos médios indica que os estados estudados estão operando com ociosidade na capacidade produtiva e que, portanto, menores custos médios ainda poderão ser alcançados. O melhor aproveitamento da capacidade produtiva da instalação, evitando a 
ociosidade produtiva, permite ao pecuarista diluir seus custos e buscar eficiência técnica, administrativa e econômica.

\section{Economias de escala (EE)}

Os indicadores de retorno à escala (RE) e de economia de escala (EE) estão apresentados na Tabela 10.

Tabela 10. Elasticidade custo, retorno à escala e economia de escala para os estados estudados, considerando o "custo da atividade leiteira" e o "centro de custo leite", agosto de 2000 a julho de 2001.

\begin{tabular}{cccc}
\hline ESTADO & ELASTICIDADE-CUSTO & RE & EE \\
\hline Custo da atividade leiteira & & \\
GO & 0,838 & 1,193 & 0,162 \\
MG & 0,975 & 1,026 & 0,025 \\
PR & 0,920 & 1,087 & 0,080 \\
RS & 0,899 & 1,112 & 0,101 \\
SP & 0,956 & 1,046 & 0,044 \\
\hline Geral & 0,907 & 1,102 & 0,093 \\
\hline GO & Centro de custos leite & & 0,163 \\
MG & 0,837 & 1,194 & 0,009 \\
PR & 0,991 & 1,009 & 0,050 \\
RS & 0,950 & 1,052 & 0,040 \\
SP & 0,960 & 1,041 & 0,029 \\
\hline Geral & 0,971 & 1,029 & 0,060 \\
\hline
\end{tabular}

Fonte: Dados da pesquisa

$\mathrm{RE}=$ Retorno à escala

$\mathrm{EE}=$ Economias de escala

Para todos os estados observaram-se valores de retorno à escala maiores que a unidade, havendo, portanto, retornos crescentes à escala, confirmando os resultados obtidos para os custos totais e custos médios. Segundo os indicadores de economias de escala (EE), que se apresentaram positivos, as empresas estudadas estão operando com tamanhos de plantas que viabilizam a obtenção de economias de escala.

O Estado de Goiás apresenta possibilidade de ganhos em relação ao aumento do volume de produção. Os estados de Minas Gerais e de São Paulo possuem os menores indicadores de retorno à escala. A análise dos indicadores de economia de escala aponta que a possibilidade de redução dos custos médios é maior quando analisados pela metodologia do "custo da atividade leiteira". 
Os indicadores empregados para a definição do perfil econômico das propriedades estudadas apontaram índices de produtividade por vaca em lactação superiores às médias de estudos anteriores, bem como à média nacional, sinalizando investimentos em insumos e manejo com vistas ao incremento da produtividade. Os indicadores econômicos também revelaram que os custos totais crescem a taxas decrescentes com o incremento da produção. As menores relações entre os custos fixos totais e os custos totais ocorreram exatamente naqueles estados com maior volume de produção.

Constatou-se que o "centro de custos leite" possui custos variáveis em uma proporção muito maior que o "custo da atividade leiteira", demonstrando uma maior especialização do subsistema leite.

Comparando-se os retornos à escala entre os estados estudados, percebe-se que o Estado de Goiás é o que apresenta maior possibilidade de ganhos em relação ao aumento do volume de produção e ao melhor aproveitamento dos fatores produtivos. O estados de Minas Gerais e de São Paulo possuem os menores indicadores de retorno à escala, indicando que estes estados encontram-se em um ponto no qual devem procurar melhorar os níveis de produtividade antes de buscarem aumentos dos níveis de produção.

\section{Conclusões}

A apuração de custos pela metodologia de "centro de custos leite" possibilitou uma maior precisão em relação às análises do custo total médio na pecuária leiteira. Pela metodologia tradicional, denominada "custo da atividade leiteira", os custos, em alguns estados, apresentaram-se significativamente diferentes. Entretanto, quando analisadas pela metodologia de "centros de custos", as diferenças entre os custos de alguns estados não foram encontradas. Isso evidencia que, no subsistema leite, a estrutura de custos daqueles estados se assemelhava, estando suas diferenças de desempenho possivelmente relacionadas aos demais subsistemas da pecuária leiteira.

A análise pela metodologia de "centro de custos leite" ainda evidenciou que, quando apurados somente os custos relacionados ao subsistema leite, os preços recebidos são suficientes para remunerar o produtor 
em todos os estados. Pela metodologia tradicional, os estados de São Paulo e do Rio Grande do Sul não apresentaram resultado econômico positivo. Dessa maneira, entende-se que a metodologia de "custos da atividade leiteira" não permite que baixos desempenhos nos demais subsistemas do sistema global de produção de leite sejam identificados.

Ficou evidente a ocorrência de ganhos com a escala de produção, tendo em vista a redução dos custos médios para maiores níveis de produção, bem como os indicadores de rendimentos à escala crescentes e de economias de escala, indicando ganhos com o crescimento da produção. Os gastos com fatores de produção resultaram em maior volume de produção. Os resultados evidenciam que os casos estudados compõemse de produtores que buscam maior eficiência produtiva e que, ainda, possuem possibilidades de ganho no que se refere a melhores combinação, alocação e aproveitamento dos recursos gerenciais e produtivos.

A análise dos indicadores de economia de escala apurados por meio das duas diferentes metodologias ainda aponta que a possibilidade de redução dos custos médios é ainda mais significativa para o sistema global de produção, ou seja, estimado pela metodologia "custo da atividade leiteira”, que para o subsistema leite, operacionalizado pela metodologia "centro de custos leite". Assim, evidencia-se, novamente, que os demais subsistemas da atividade global têm apresentado um desempenho inferior quanto ao aproveitamento dos recursos produtivos e gerenciais em relação ao subsistema de produção de leite. Isto também indica que as ações em relação à extensão e às políticas públicas devem atentar para a capacitação e profissionalização dos demais subsistemas da pecuária leiteira, bem como para o aperfeiçoamento da capacidade gerencial do setor produtivo.

\section{Referências bibliográficas}

ALENCAR, E.; GRANDI, D. S.; ANDRADE, D. M.; ANDRADE, M. P. de. Complexos agroindustriais, cooperativas e gestão. Organizações Rurais e Agroindustriais, Lavras, v. 3, n. 2, p. 30-44, jul./dez. 2001.

FASSIO, L. H.; REIS R. P.; YAMAGUCHI, L. C. T.; REIS, A. J. Custos e shutdown point da atividade leiteira em Minas Gerais. Revista de Economia e Sociologia Rural, Brasília, v. 43, n. 4, p. 759-777, out./dez. 2005. 
GASTAL, E. Enfoque de sistemas na programação da pesquisa agropecuária. Rio de Janeiro: IICA, 1980. 207p.

MARQUES, V. M.; REIS, R. P.; SÁFADI, T.; REIS, A. J. dos. Custos e escala na pecuária leiteira: estudo de casos em Minas Gerais. Ciência e Agrotecnologia, Lavras, v. 26 n. 5, p. 1027-1034, set./out. 2002.

REIS, R. P. Fundamentos de economia aplicada. Lavras: UFLA/FAEPE, 2002. 95p.

REIS, R. P.; MEDEIROS, A. L. e MONTEIRO, L. A. Custo de Produção da Atividade Leiteira na Região Sul de Minas Gerais. Organizações Rurais e Agroindustriais, Lavras, v. 3, n. 2, p. 45-54, jul./dez. 2001.

SERVIÇO DE APOIO ÀS MICRO E PEQUENAS EMPRESAS DE MINAS GERAIS. FEDERAÇÃO DA AGRICULTURA DO ESTADO DE MINAS GERAIS. Relatório da pecuária leiteira do Estado de Minas Gerais. Belo Horizonte, 1996. 2v.

TUPY, O.; ALVES, E. R. de A.; ESTEVES, S. N.; SCHIFFLER, E. A. Método para controle e análise de custo de produção de leite. São Carlos: EMBRAPA PECUÁRIA SUDESTE, 2000. 35p. (Circular Técnica, 26)

YAMAGUCHI, L. C. T. Custo de Produção do Leite: um novo enfoque. Boletim do Leite, Piracicaba, v.7. n.76, p. 1-2, jul. 2000.

YAMAGUCHI, L. C. T.; MARTINS, P. do C.; CARNEIRO, A. V. Produção de leite no Brasil nas três últimas décadas. In: GOMES, A. T.; LEITE, J. L. B.; CARNEIRO, A. V. (Ed.). O agronegócio do leite no Brasil. Juiz de Fora: EMBRAPA/CNPGL, 2001. p. 33-48

YAMAGUCHI, L. C. T.; MARTINS, P. do C.; CARNEIRO, A. V.; MACHADO, A. D. C. Custo de produção do leite: abrindo a caixa preta. Curvelo: Cooperativa Agropecuária de Curvelo. EMBRAPA/CNPGL, 2002. 72p.

Recebido em novembro de 2006 e revisto em abril de 2007. 